\title{
CYP3A5 contributes to PDAC chemoresistance
}

New research sheds light on the mechanisms underlying resistance to chemotherapeutics in patients with pancreatic ductal adenocarcinoma (PDAC). Increased expression of CYP3A5, which metabolizes the cytoskeleton-targetting drug paclitaxel and the tyrosine-kinase inhibitor erlotinib, contributes to tumour-cell-autonomous and acquired drug resistance in different PDAC subtypes.

PDAC is an aggressive cancer with a poor prognosis. Current chemotherapy drugs produce limited survival benefit, in part because PDAC tumours are intrinsically resistant or acquire resistance to treatment. "Although some progress has been made in recent years, the underlying mechanisms of treatment resistance in PDAC remain poorly understood," says author Martin Sprick. "Our first goal was to develop improved model systems which closer resemble patient tumours."

Using patient-derived PDAC tumour samples xenografted on to the pancreata of immunodeficient mice, the researchers first generated primary PDAC cell lines. Whereas only two of the three tumour subtypes (exocrine-like, quasi-mesenchymal (QM) and classical) found in patient tumour samples were represented in previously available PDAC cell lines, the primary cell lines derived by the authors contained all PDAC subtypes.

To assess whether PDAC subtypes differ in their sensitivity to chemotherapy agents, the authors treated the patient-derived PDAC cell lines with paclitaxel or erlotinib. The exocrine-like tumour subtype was markedly more resistant to these drugs than other subtypes - subsequent analysis revealed increased expression of CYP-family enzymes involved in drug metabolism in exocrine-like tumour cells. "Although expression of CYP-family enzymes has been described for various tumours, to our knowledge no one has demonstrated that tumours can acquire this mechanism to locally inactive drugs," explains author Elisa Noll. CYP3A5 was highly expressed exclusively in exocrine-like PDAC tumour cells; knockdown of this enzyme rendered these cells sensitive to erlotinib and paclitaxel. Furthermore, mice with established exocrine-like tumours in which CYP3A5 expression was ablated were more sensitive to erlotinib and paclitaxel than mice with tumours expressing CYP3A5.

The effectiveness of smallmolecule drugs in PDAC is also limited by acquired resistance. Notably, CYP3A5 was upregulated by

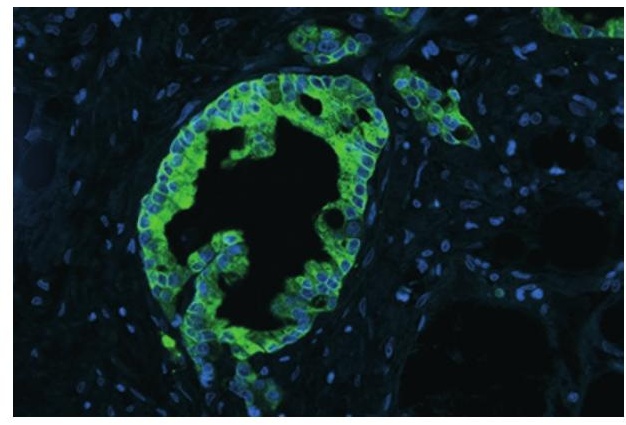

Patient PDAC biopsy sample stained for CYP3A5 (green) and nuclei (blue). Courtesy of E. M. Noll.

treatment with paclitaxel or erlotinib in xenograft tumours that were previously CYP3A5-negative. The researchers also showed that acquired resistance to erlotinib and paclitaxel in classical-subtype and QM-subtype cell lines was associated with induced CYP3A5 expression. Knockdown of CYP3A5 in these cell lines restored drug sensitivity.

"Developing approaches to inhibit the CYP3A5 enyzmatic cascade could pave the way to overcoming drug resistance in a subset of patients with PDAC," concludes author Andreas Trumpp.

Hugh Thomas

ORIGINAL ARTICLE Noll, E. M. et al. CYP3A5 mediates basal and acquired therapy resistance in different subtypes of pancreatic ductal adenocarcinoma. Nat.Med. http://dx.doi.org/ 10.1038/nm.4038 